\title{
IMPORTANCIA DE LA FUNCIÓN ACADÉMICA EN LA RSU. PROPUESTA DE UN "PROGRAMA DE SENSIBILIZACIÓN”
}




\title{
RESUIMEN
}

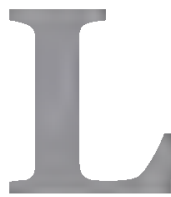

a Responsabilidad Social Universitaria (RSU) es una política de mejora continua de la Universidad hacia el cumplimiento efectivo de su misión social mediante 4 procesos: Gestión ética y ambiental de la institución; Formación de ciudadanos conscientes y solidarios; Producción y Difusión de conocimientos socialmente pertinentes; Participación social en promoción de un Desarrollo más equitativo y sostenible. (VALLAEYS, F., 2008).

Para comenzar a gestionar la RS es necesario adoptar un modelo que comienza por comprenderla. La función académica, en tal sentido, es clave en la universidad, toda vez que a través de ella se produce la formación de los egresados tanto científica como deontológica. Resulta por ello necesario incluir en las currículas y programas de las asignaturas de las distintas carreras que conforman la Universidad Nacional del Nordeste (UNNE) -de manera trasversal-contenidos relativos a la ética y la responsabilidad social en el actuar del profesional. A tal fin se propone un programa de formación, a través de un Seminario - Taller para los docentes de esta casa de altos estudios, con el objeto de sensibilizarlos en lo que a RSU se refiere.

Palabras Clave: Mejora Continua - Ética aplicada - Universidad.

\begin{abstract}
"The Universitary Social Responsibility (USR) is a policy of continuous improvement of the University towards the effective fulfillment of its social mission by means of 4 processes: Ethical and environmental management of the institution; Formation of conscious and shared in common citizens; Production and Diffusion of socially pertinent knowledge; Social Participation in promotion of a more equitable and sustainable Development". (VALLAEYS, F., 2008).

In order to begin to manage SR it is necessary to adopt a model that begins by including understand it. The academic function, in such sense, is essential in the university, every time through its formation of withdrawn as much the scientist as deontologic issues take place. It is, for that reason, necessary to include in profiles and programs of the subjets of the different proffesions that conform University National of Northeast (UNNE) - on trasversal way - containts relative to the Ethics and the Social Responsibility in acting of the professional. To such aim a formation program sets out, through a Seminary - Workshop for the professors of this house of high studies, with the intention of sensitizing them in what USR talks about.
\end{abstract}

Keywords: Continuous improvement - Ethical applied - University. 


\section{INTRODUCCIÓN}

La Responsabilidad Social Universitaria (RSU) es una manera de gestionar las Universidades que deriva de la plena convicción de las autoridades de tales instituciones y -para considerarse tal- debe estar integrada a la administración de cada una de las partes que la componen.

El nuevo slogan de la UNNE reza: "Gestión para la Excelencia con Responsabilidad Social e Innovación”, pero, en la actualidad, lo cierto es que si bien se llevan a cabo diversas iniciativas que podrían tener que ver con la filosofía de RS en la Universidad Nacional del Nordeste (en adelante UNNE), dichos emprendimientos se realizan de manera aislada, atomizada y no existe una correcta comunicación a los distintos actores que formamos parte de esta Casa de Estudios acerca de las medidas que se proyectan ni que se implementan.

Aportar soluciones al problema de la RS trasciende el marco estrictamente académico, pero la realización de "ajustes" desde la Educación Superior tiene una relevancia indiscutible, puesto que es uno de los ámbitos naturales para liderar los cambios que llevan a una evolución continua y estrechamente relacionada con los requerimientos del mundo científico y profesional.

Según François Vallaeys (2008) "La Responsabilidad Social Universitaria es una política de mejora continua de la Universidad hacia el cumplimiento efectivo de su misión social mediante 4 procesos: Gestión ética y ambiental de la institución; Formación de ciudadanos conscientes y solidarios; Producción y Difusión de conocimientos socialmente pertinentes; Participación social en promoción de un Desarrollo más equitativo y sostenible".

Es decir que la RSU supone la asunción por parte de la Universidad de la responsabilidad de gestionar de manera ética y transparente y en estrecha relación con la sociedad en la que se desempeña las cuatro funciones básicas de su misión: académica, investigación, transferencia y gestión, a través de estrategias específicas.

"La responsabilidad social universitaria implica ofertar servicios educativos y transferencia de conocimientos siguiendo principios de ética, buen gobierno, respeto al medio-ambiente, compromiso social y promoción de valores ciudadanos, responsabilizándose así de las consecuencias y los impactos que se derivan de sus acciones”. (DE LA CUESTA GONZÁLEZ, M., DE LA CRUZ AYUSO, C., RODRÍGUEZ FERNÁNDEZ, J., 2010).

El desarrollo de estas estrategias, debería realizarse a través de la participación integrada de los grupos de interés internos y externos en el cometido de la Universidad; la articulación de los planes de estudios, la investigación, la extensión y los métodos de enseñanza con la solución de los problemas de la sociedad; el autodiagnóstico regular de la institución con herramientas apropiadas de medición y comunicación para la rendición de cuentas hacia los grupos de interés (Cfr. VALLAEYS, F., DE LA CRUZ, C. y SASIA, P., 2009).

En este trabajo, proponemos el análisis de una estrategia de mejora de la RSU a través de la función académica, mediante la sensibilización del plantel docente acerca de la importancia de este modelo de gestión - transferencia - investigación - enseñanza de cara a la articulación de contenidos curriculares y la solución de problemas sociales. 


\section{BREVE RESEÑA DE LA RS EN LA UNNE}

La UNNE funciona en las Provincias de Chaco y Corrientes y está compuesta por once Facultades, algunas carreras dependientes de ellas; tres carreras dependientes del Rectorado y "extensiones áulicas" en las que se propende la modalidad de educación semipresencial, siendo las más importantes de ellas -en la Provincia del Chaco- las de General Pinedo y Juan José Castelli y -en Corrientes- las de Paso de los Libres y Goya.

Esta dispersión geográfica complica -de cierto modo- la difusión de los programas que se realizan en cada Unidad Académica y dificulta la actuación de la Universidad en su conjunto, así como la transversalidad de las temáticas.

A continuación se reseñan algunas iniciativas de RS que se han venido desarrollando en esta universidad aunque, desde ya, adelantamos que se trata de iniciativas aisladas y no de políticas integrales ni trasversales como la gestión de la RSU requiere:

\subsection{AGENTIA. (AGENTIA, 2010)}

La Agencia PyMe de la UNNE, es una unidad operativa dependiente de Rectorado, articulada transversalmente a las Secretarías Generales, a las Unidades Académicas y a otras Unidades Organizacionales.

Tiene como misión mejorar la calidad de vida de los habitantes de la región en forma creciente y sustentable promoviendo el espíritu emprendedor como modelo de rol para el cambio social.

En aras de esa finalidad, Agentia puede asumir los roles de facilitador, tutor, multiplicador, consultor, capacitador o especialista con la participación de docentes, alumnos, graduados y no docentes, en equipos interdisciplinarios, tendientes a articular acciones para fortalecer la calidad, la excelencia, la pertinencia y el desarrollo sustentable de la Región.

Presta servicios de asistencia técnica para empresas y emprendedores, realiza estudios de proyectos de empresas (viabilidad del proyecto), realiza diagnósticos organizacionales (midiendo factores como el clima laboral, desempeño y productividad), promueve el asociativismo para la colaboración entre empresas, asesora en cuanto al acceso a líneas de financiamiento, colabora en la formulación y evaluación de proyectos y realiza tareas de capacitación de diferentes clases (programas generales, enfocados e in company).

\subsection{Centro de Gestión Ambiental y Ecología (CEGAE) (CEGAE, 2010).}

Es un Instituto dependiente de Rectorado; se encamina hacia una apertura de la comunidad universitaria hacia la sociedad civil, mediante la vinculación y extensión de las actividades generadas en la Universidad en el tema ambiental en el marco de una relación sustentable entre la educación, el trabajo y el aprovechamiento racional de los recursos naturales dentro de las nuevas políticas globales de desarrollo sustentable; siendo sus principales objetivos:

* Consolidar una cultura y una conciencia popular participativa, tendiente a una armónica interacción con el ambiente, buscando una mejor calidad de vida. 
* Propender a la formación de los alumnos y docentes de la universidad en una concepción conservacionista dentro del marco de las nuevas políticas globales de desarrollo sustentable.

* Vinculación con organismos e instituciones nacionales y extranjeras que se encuentren abocados a la implementación de una gestión ambiental.

Para el cumplimiento de tales objetivos se ha creado el Sistema de Gestión Ambiental (SIGEA) que desarrolló un atlas ambiental de las provincias de Chaco y Corrientes, descriptivo de los distintos medios naturales (agua, tierra, aire, flora, fauna y paisajes), así como los medios sociales (comprensivo de los aspectos sociales, político - administrativos y de infraestructura). También se describen las normativas ambientales y los respectivos indicadores de la región.

También en el marco del CEGAE se ha publicado el "Manual de Biodiversidad de Chaco, Corrientes y Formosa" que tiende a brindar la información necesaria para conocer y valorar los recursos naturales.

Este Instituto también gestiona el programa Ecocampus UNNE que se desarrolla en el Campus Resistencia de esta Universidad. Se presenta como una oportunidad para mejorar las condiciones ambientales e inducir al cambio de actitudes y comportamientos de toda la población universitaria, introduciendo la dimensión ambiental en la gestión de sus edificios, la planificación, ordenación del mismo y el uso racional de los recursos a través de un proceso participativo para la búsqueda de soluciones que propicien la mejora ambiental de su entorno.

Las líneas de acción propuestas son las siguientes:

1.- Transporte sostenible.

2.- Diseño y ordenación del campus.

3.- Gestión y minimización de residuos.

4.- Gestión y ahorro del agua.

5.- Calidad del aire.

6.- Eficiencia energética.

7.- Criterios ambientales en compras y contratos.

8.- Indicadores.

9.- Control y confort ambiental.

10.- Gestión de áreas verdes.

11.- Educación y sensibilización ambiental.

En el área de educación, el CEGAE ha implementado dos programas: Ambientación Curricular (en las Facultades de Ingeniería y Medicina) y Cátedra Libre de de Educación para el Desarrollo Sostenible, con el propósito de abrir un espacio de reflexión y desarrollo del tratamiento de las problemáticas ambientales, mediante la creación de una plataforma académica para el desarrollo de actividades de formación, capacitación y extensión, que permitan el tratamiento de estos temas que se presentan en el mundo actual desde una perspectiva transversal a las distintas carreras de la Universidad y a la comunidad en general y que contemple su 
abordaje en términos de integridad ambiental, viabilidad económica y una sociedad justa para las generaciones presentes y futuras. En este marco, se propone la realización de distintas instancias de formación, intercambio, orientación y difusión de los conocimientos en Educación para el Desarrollo Sostenible, por medio de conferencias, cursos, talleres, etc., a ser desarrollados en formato virtual, semipresencial o presencial, según correspondiere.

Asimismo, se plantea la implementación y el dictado de una Asignatura: Educación Ambiental, que contemple la posibilidad de ser incluida como materia optativa en la currícula de las distintas carreras de la UNNE. La Asignatura será implementada en modalidad virtual, a través de la UNNE Virtual. Para ello se contará con la colaboración de la Universidad de Valencia, España.

\subsection{Centro de Estudios Sociales (CES) (CES, 2010).}

Es un Instituto dependiente de Rectorado cuya actividad científica se orienta en el campo de las Ciencias Sociales con la pretensión de generar un ámbito de reflexión teórica vinculado, al mismo tiempo, con los problemas de la región. Su fin principal es contribuir a la consolidación de un espacio institucional propio para las Ciencias Sociales en la región Nordeste. Sus actividades están dirigidas a las siguientes líneas de acción:

* Investigaciones disciplinares, transdisciplinares y de investigaciónacción-participativa. Formación de Investigadores.

* Promoción y realización de estudios significativos en relación con el desarrollo de la región.

* Desarrollo de estrategias de intervención social que contribuyan a consolidar procesos de promoción comunitaria y construcción de ciudadanía.

Sus líneas de investigación son:

- Desarrollo social: Procesos de inclusión/exclusión, políticas públicas, procesos de participación y construcción de ciudadanía: Examina distintos procesos sociales vinculados con las asimetrías del desarrollo, las formas que adquieren, la incidencia en las condiciones de vida, la construcción de las identidades y de la ciudadanía.

- Estudios sobre el Mercado laboral: Investiga los modos en que se estructuran y modifican los mercados laborales y las formas de inserción que presentan los distintos colectivos.

- Estudios sobre representaciones sociales e imaginarios sociales: Se trata de una línea de investigación transversal, en tanto modelo de análisis que permite el abordaje de los procesos de simbolización y la construcción de la realidad.

- Educación y Sociedad: Considera los aspectos sociales, culturales y políticos que atraviesan y definen la situación educativa.

- Investigación acción: Vinculada con el desarrollo de experiencias de intervención en el marco de la Responsabilidad Social Universitaria.

Este instituto también ha desarrollado Cátedras Libres, como ser: Cátedra Libre UNNEOEI "Ética, Ciudadanía y ejercicio Profesional"; "Cátedra ACNUR: Universidad y Derechos Humanos" y "Cátedra de Ciencias Sociales de la UNNE". Asimismo, el Centro de Estudios 
Sociales es miembro de diversas Redes que promueven la formación de capital social.

\section{EXPERIENCIAS DE RSU EN LA FACULTAD DE CIENCIAS ECONÓMICAS DE LA UNNE}

Teniendo en cuenta que este trabajo se presenta en oportunidad de la celebración del 60 aniversario de la Facultad de Ciencias Económicas de la UNNE, consideramos acertado enunciar algunas iniciativas de RSU que se han realizado y se están realizando, particularmente en esta casa de estudios: el Programa de Voluntariado Universitario y el Programa de Ética para el desarrollo.

\subsection{El "Programa de Voluntariado Universitario". (República Argentina,} Ministerio de Educación, 2010)

El programa de Voluntariado Universitario es impulsado y financiado por la Secretaría de Políticas Universitarias del Ministerio de Educación de la Nación Argentina.

La Facultad de Ciencias Económicas de la UNNE es una de las Unidades Ejecutoras de este Programa. Este promueve la vinculación de las distintas instituciones miembro con la comunidad en que se insertan, a través de propuestas orientadas a mejorar la calidad de vida de su población. Se propone incentivar el compromiso de los estudiantes de nivel superior universitario con el medio social, promoviendo su participación en la construcción de una sociedad inclusiva.

Desde su creación en 2006, el Programa invita a estudiantes y docentes de universidades a presentar propuestas destinadas a atender a los sectores que más lo necesitan. El objetivo fundamental es fomentar y promover la función social de la universidad.

Las iniciativas están relacionadas con alguno de los ejes requeridos: la reinserción escolar, la retención escolar, el acompañamiento a las trayectorias de los alumnos y la orientación para el ingreso a la universidad.

Los Proyectos de la Facultad de Ciencias Económicas de la UNNE se denominaron " $\mathrm{Ca}$ pacitación en procesos de gestión empresarial y participación para emprendedores" (ZARATE, J., CRUZ DE INNOCENTE, R., DE CASTRO, I., JEREZ, S., FRANCHINI, N. y GUALTIERI, J., 2006 Y CRUZ DE INNOCENTE, R., FRANCHINI, N. JEREZ, S., DE CASTRO, I., GUALTIERI, D., ZÁRATE, J. ET AL., 2007).

Fueron llevados a cabo por la Facultad de Ciencias Económicas de la UNNE en los años 2007, en la ciudad de Barranqueras, y 2008 en la ciudad de Resistencia, en colaboración con sus respectivas Municipalidades, y ejecutados por equipos docentes y de alumnos de esta Casa de Estudios.

Cabe destacar que la UNNE está inserta geográficamente en el área de menor desarrollo relativo de nuestro país; con indicadores que colocan a dicha área como la de más bajo Índice de Desarrollo Humano (IDH) e ingreso por habitante, la de menor desarrollo educativo, la de más baja tasa de escolaridad, la de mayor desgranamiento escolar, con altas tasas de analfabetismo, el más bajo porcentaje de educación en áreas rurales, preocupantes tasas de desempleo y las menores tasas de expectativas de vida de su población.

En la implementación de los Proyectos, se desarrollaron tareas de capacitación para mi- 
croemprendedores provenientes de ambas ciudades en relación a la gestión de empresas en general, organización administrativa yjurídica, regímenes tributarios aplicables a los distintos emprendimientos, formulación y evaluación de proyectos, financiamiento y acceso al crédito, ayudas gubernamentales, marketing y herramientas informáticas básicas.

Estas tareas fueron, luego, evaluadas -a los alumnos- en cuanto a los contenidos, y a las tareas de capacitación propiamente dichas, mediante encuestas; para, finalmente, entregar los respectivos certificados de la capacitación impartida.

Para la primera ejecución del proyecto, en la ciudad de Barranqueras, participaron 320 microemprendedores, 16 alumnos de las distintas carreras de la Facultad de Ciencias Económicas y 6 docentes, también de esta Casa de Estudios. Para la segunda ejecución, en la ciudad de Resistencia, se capacitaron 140 emprendedores, participando en la capacitación 20 alumnos y 11 docentes. Teniendo en cuenta los objetivos planteados originalmente en la elaboración del Proyecto, y en función de las actividades desplegadas, se ha logrado que los microemprendedores:

a.- Desarrollen habilidades para diferenciar los distintos tipos de organizaciones y emprendimientos en su funcionamiento: La meta alcanzada fue que los destinatarios están mejor preparados para seleccionar la mejor alternativa (legal, administrativa, impositiva, etc.) posible para su proyecto empresarial emprendedor. Los indicadores de resultados fueron la realización de tareas de investigación sencillas y de rutina en el emprendimiento propio y los resultados de evaluaciones periódicas que se realizaron.

b.- Manejen las distintas alternativas estratégicas que se le pueden presentar, para insertarse con un emprendimiento propio: La meta alcanzada fue que los micro emprendedores están capacitados para identificar las distintas herramientas de gestión y pueden elegir la o las más adecuadas a su emprendimiento, siendo indicador de este resultado la participación activa de los emprendedores en los diversos talleres realizados.

c.- Identifiquen los distintos requerimientos en materia impositiva, tributaria y previsional que son requeridos por los organismos del Estado Nacional, Provincial y Municipal. Metas e indicadores iguales a los del ítem 1.-

d.- Desarrollen técnicas y habilidades básicas para el uso de herramientas informáticas (equipos y programas) en la administración de una organización: La meta alcanzada fue que los destinatarios están capacitados para el uso de aplicativos básicos de una PC y el indicador de resultados fue la elaboración de documentos sencillos en Word y Excel.

e.- Conozcan y desarrollen habilidades para manejar herramientas básicas de gestión, útiles y necesarias para la toma de decisiones. Metas e indicadores iguales a los del ítem 2.-

Los objetivos específicos planteados en relación a los aprendizajes de los estudiantes fueron los siguientes: Lograr que los alumnos:

1.- Se integren a la comunidad en una práctica profesional anticipada.

2.- Articulen contenidos académicos con acciones solidarias que permitan la formación de profesionales comprometidos con las necesidades y problemáticas comunitarias locales.

3.- Desarrollen el compromiso social como futuros profesionales a través de la compren- 
sión de la función social del conocimiento.

4.- Participen en la identificación y solución de problemáticas comunitarias. Las metas alcanzadas son que los estudiantes que protagonizaron el programa de voluntariado:

* Tomaron conciencia de este nuevo paradigma del aprendizaje - servicio.

* Articularon contenidos académicos con acciones solidarias eficaces.

* Se pusieron al servicio de necesidades y demandas sociales específicas y promovieron la inserción comunitaria de las instituciones educativas como instrumento eficaz para superar las problemáticas y necesidades locales.

* Este grupo de estudiantes devolvió - con esta práctica educativa solidaria- a la comunidad, lo que recibieron de ella, por medio de los conocimientos que adquirieron en una carrera universitaria, comprometiéndose con el entorno social, económico y productivo de la región.

\section{2. "Programa Nacional de Formación en Ética para el Desarrollo. Premio AMARTYA SEN"}

Este programa está destinado a formar a los graduados jóvenes más destacados y a estudiantes del último año de las carreras que se dictan en la Facultad, en las ideas más avanzadas a nivel internacional sobre un desarrollo con ética. Apuesta, asimismo, a la formación de nuevas generaciones de docentes que contribuyan a una visión ética y de responsabilidad social corporativa de la economía y de la gerencia.

Es conducido académicamente por el Dr. Bernardo Kliksberg, Profesor Honorario de la Universidad de Buenos Aires (UBA), y tiene el propósito de "tratar de convencer al mundo y a los hacedores de la política económica que el desarrollo es mucho más que el crecimiento económico, porque debe tener como objetivo principal el bienestar de la gente".

$\mathrm{Al}$ respecto, se ha firmado un convenio entre el Ministerio de Educación, el Ministerio de Economía y Finanzas Públicas de la Nación y la Universidad de Buenos Aires para poner en marcha una nueva edición, cuya administración estará a cargo de esta última y en la que participan otras Facultades de Ciencias Económicas de Universidades Públicas como la del Nordeste.

El programa enseña a enseñar áreas como responsabilidad social empresarial, capital social, ética para el desarrollo, gerencia social y otras. Los jóvenes formados, multiplican después esos conocimientos enseñando en las aulas o en programas de voluntariado e inclusión social.

Puntualmente, la manifestación de este programa en la Facultad de Ciencias Económicas de la UNNE se está realizando a través de jornadas de capacitación destinada a los jóvenes profesionales y alumnos involucrados en el proyecto, pero abiertas al público en general, debido a la trascendencia de los temas que se tratan en estas jornadas.

A la fecha se han realizado siete Conferencias sobre las siguientes temáticas: Una visión holística del desarrollo y las ciencias gerenciales, Capital Social, Responsabilidad Social Empresaria (2 charlas), Responsabilidad Social Universitaria, Voluntariado Universitario y Gerencia Social, quedando pendientes las siguientes: Derechos Humanos y Derechos Colectivos, 
Sustentabilidad Ambiental / Desarrollo Sostenible, Economía Social y Desarrollo Social, Economía Social y Solidaria y Desarrollo Social, Economía Social y Solidaria experiencias locales: Asociación Civil Lapacho y Consumo Responsable.

A estas charlas se le suman capacitaciones específicas para los participantes del programa para formarlos en la elaboración y aplicación de un proyecto social que deberán desarrollar e implementar.

\section{RSU Y LA FUNCIÓN ACADÉMICA}

El desarrollo de RS requiere la participación en estructuras sociales, siendo la Universidad, por su función de formación, un espacio ideal para la atención y desarrollo de acciones que promuevan el diálogo, los valores de juicio moral y la identificación de alternativas pro sociales aplicadas al desarrollo sostenible.

VALLAEYS, F., DE LA CRUZ, C. y SASIA, P. (2009) en su Manual de Primeros Pasos en Responsabilidad Social Universitaria, proponen un modelo de cuatro etapas para la implementación del sistema de la RSU consistentes en:

1.- Comprender, Convencer y Comprometer la universidad para la RSU;

2.- Realizar un autodiagnóstico participativo;

3.- Contrastar los resultados de este diagnóstico con el compromiso misional de la universidad;

4.- Evaluar y comunicar de forma transparente los resultados de todos los proyectos de mejora institucional.

Para comenzar con la gestión de la RSU en la UNNE, deberíamos entonces iniciar este proceso de mejora continua por el primer paso, aclarando y comunicando en qué consiste la RSU, para poner de acuerdo a los distintos miembros de la comunidad universitaria acerca del compromiso social de esta casa de estudios y lo que significa para cada uno; garantizando, desde las máximas autoridades los medios para poder cumplir efectivamente con la política de Responsabilización Social Institucional.

"Hablamos de "responsabilización" porque entendemos la dinámica de la RSU no como modelo acabado para copiar y replicar fidedignamente, sino como un horizonte de sentido, una brújula y un estímulo, incluso como una inquietud institucional permanente para la mejora continua de la Universidad hacia poder asumir su compromiso social, sin poder nunca pretender haber cumplido totalmente con la meta, lo que no sería más que presunción y sospecha de autosatisfacción". (VALLAEYS, F., DE LA CRUZ, C. y SASIA, P., 2009).

El presente pretende entonces ofrecer a quienes se encargan de la formación de los alumnos, herramientas básicas acerca de lo que significa la RSU, la finalidad del modelo en el marco de la pertinencia de los contenidos de las currículas de las carreras universitarias y la propuesta de implementación de contenidos éticos de manera trasversal en las distintas asignaturas que se dictan en la Facultad de Ciencias Económicas, para -en una etapa posterior- extender- 
las al resto de las unidades académicas de la UNNE.

Durante la formación universitaria, además de aportarse aprendizajes cognitivos y técnicos, se debería formar en "en la capacidad de comprometerse, de escucha y de diálogo, de tomar distancia ante los problemas, de saber mirar a través de los ojos del otro, de aprender a ponerse en su lugar, de tener un pensamiento crítico (...). Formar personas comprometidas con su entorno y con sus semejantes, desde el reconocimiento de que su acción como profesional no solo tiene repercusiones en su entorno más inmediato, sino que va mucho más allá en el espacio y el tiempo (DE LA CALLE, C., GARCÍA RAMOS, J. M. Y GIMÉNEZ, P., 2007).

La idea rectora de esta propuesta es que la formación profesional y humanística se oriente realmente hacia un egresado que haya logrado e incorporado competencias ciudadanas de RS para el desarrollo sostenible de su comunidad. "Esto implica que la orientación curricular general, y parte de los cursos de cada carrera, tengan una relación estrecha con los problemas reales de desarrollo (económicos, sociales, ecológicos ...) y puedan dictarse in situ en contacto con actores externos, bajo el método del Aprendizaje basado en Proyectos Sociales (Aprendizaje - Servicio)". (VALLAEYS, F., DE LA CRUZ, C. y SASIA, P., 2009).

Esta idea se propone en busca de una universidad centrada en contenidos, actitudes y valores en busca de un equilibrio entre la excelencia académica y la formación humanista en valores para formar profesionales, pero también ciudadanos con principios éticos y valores solidarios. "Que sea capaz de ver las necesidades de la sociedad en su conjunto y dar solución a sus problemas. Que contribuya al bien colectivo, la construcción social y el desarrollo humano. Que forme ciudadanos participativos y que se involucre en actividades cívicas relevantes en las comunidades en las que opera y en el ámbito internacional" (DE LA CUESTA GONZÁLEZ, M., DE LA CRUZ AYUSO, C., RODRÍGUEZ FERNÁNDEZ, J., 2010).

Más precisamente, en el aspecto formativo en el que estamos enfocando este proceso implica incorporar en las currículas el diseño de aspectos relativos a la ética y deontología profesional, a la vez que incentivar la toma de conciencia sobre la construcción social y el impacto de las tecnologías en términos de sustentabilidad económica, utilidad social y cuidado del medioambiente. "Junto con ello, se requiere garantizar con evidencias y resultados que en los planes de estudios los alumnos adquieran competencias apropiadas en materia de igualdad, discapacidad, derechos humanos, solidaridad, ética de los negocios, sostenibilidad y cooperación al desarrollo de los pueblos" (DE LA CUESTA GONZÁLEZ, M., DE LA CRUZ AYUSO, C., RODRÍGUEZ FERNÁNDEZ, J., 2010).

Es que la universidad es la encargada de producir y difundir un "conocimiento socialmente responsable", entendiéndose por tal "aquel al cual se le cualifica por su soporte científico y técnico, con suficiente bagaje teórico y con aplicabilidad social; un conocimiento con capacidad de aportar al mejoramiento de las condiciones de vida y a la dignificación de las dolorosas circunstancias que golpean a un buen número de congéneres que conviven entre nosotros, junto a nosotros" (MARTÍNEZ, R., 2008). 


\section{PROPUESTA DE UN SEMINARIO DE SENSIBILIZACION SOBRE LA RSU}

En virtud de todo lo expuesto es que consideramos necesario iniciar el proceso de responsabilización social de la Universidad Nacional del Nordeste con el planteamiento de un Seminario - Taller para sensibilizar a los docentes de esta casa de estudios acerca de los contenidos, procedimientos y valores que implica la RSU.

\subsection{Fundamentación}

Las corrientes sobre Responsabilidad Social surgen, primeramente, en el campo de las empresas, algunos autores como Peter Drucker piensan que nacen como un requerimiento de los ciudadanos a un nuevo grupo de poder, ante las ineficiencias del Estado para resolver los "grandes problemas".

Hoy en día existen diversas organizaciones que se dedican al estudio y la difusión de la Responsabilidad Social Empresaria y es profusa la bibliografía sobre este tema, aunque el viraje que los tiempos requieren se está llevando su calificativo de "Empresaria" para dejar a la Responsabilidad Social "sin apellido". Esto significa que se requiere un actuar socialmente responsable tanto de las empresas, cuanto de los gobiernos, de las organizaciones de la sociedad civil y de los ciudadanos.

Las Universidades no quedan exentas de esta exigencia, cuando las urgencias éticas de nuestro planeta nos piden otra forma de pensar y una práctica distinta para crear una realidad diferente.

Establece el artículo $2^{\circ}$ del Estatuto de nuestra Universidad que la misma: “...Procura formar integral e interdisciplinariamente a sus componentes e infundir en ellos el espíritu crítico y a la vez el sentido de responsabilidad personal y social de reafirmación ético cívica y de sus convicciones democráticas y republicanas, dentro de un marco de pluralidad y respetuoso disenso".

Esta visión impone la necesidad de repensar nuestras propuestas académicas, de extensión, de investigación y, sobre todo, de gestión extendiendo la acción y servicios universitarios a la Comunidad a la que ésta pertenece, en un clima de libertad, justicia, igualdad y solidaridad, tal como lo propone su finalidad primordial.

\subsection{Destinatarios}

Los participantes serán profesores de la Universidad Nacional del Nordeste, interesados en incluir en sus tareas de enseñanza, investigación, extensión y gestión las nociones de Responsabilidad Social Empresaria, Gubernamental, de las Organizaciones de la Sociedad Civil y Ciudadana.

\subsection{Objetivos}

En función de los fundamentos expuestos, las metas propuestas para el cursante son las siguientes: 
* Sensibilizarlos acerca del significado de la Responsabilidad Social en un contexto de visión sistémica de las organizaciones y dentro de la cultura de las mismas.

* Incorporar los conceptos básicos de la Responsabilidad Social Universitaria, como sustrato ético, en los programas de estudio, planificación de carreras, actividades de extensión, proyectos de investigación y en el comportamiento cotidiano de los participantes en relación a todos los grupos de interés, tanto propios de la Universidad, cuanto externos.

* Establecer la concepción de la RSU como marco transversal de la formación de cualquier profesional que egrese de esta casa de estudios.

* Proponer ideas que involucren a los interesados en proyectos de RSU.

\subsection{Contenido UNIDAD I:}

1.- Responsabilidad Social. Concepto y evolución histórica.

2.- Teoría de los Sistemas y Teoría de los Stakeholders: su importancia en el análisis científico y organizacional.

3.- Gestión Estratégica: definiciones de: visión, misión, objetivos y operaciones de la organización.

Objetivos Específicos:

* Comprender la evolución del concepto de Responsabilidad Social desde la década del '50 hasta la actualidad.

* Relacionar esta evolución con los escándalos provenientes de falencias éticas en los mercados, tales como Enron y World Com, así como la crisis generada por la "burbuja financiera" que aún surte efectos en la economía mundial.

"Incorporar la Teoría de los Sistemas como modelo de análisis científico.

* Estudiar la Teoría de los Stakeholders como técnica de análisis, planificación, implementación y control de las organizaciones.

* Comprender qué son los grupos de interés de una determinada organización y analizarlos -en particular- respecto al ámbito universitario. Comprender las definiciones esenciales de la estrategia de una organización.

\section{UNIDAD II:}

1.- Ética aplicada en las organizaciones: ¿De quién se requiere un actuar ético? Ética como filosofía moral, ética aplicada, ética cívica.

2.-. Cultura Empresarial: los valores que implica una gestión de la Responsabilidad Social.

3.- Implementación de la Ética en la Gestión: Desde adentro hacia afuera y desde afuera hacia adentro.

Objetivos Específicos:

* Entender la relevancia de un actuar ético en y desde las organizaciones. 
* Analizar las formas de implementación de la Responsabilidad Social como estrategia desde y hacia las organizaciones.

* Analizar los valores que comprende la Responsabilidad Social como origen de una nueva ética basada en el cuidado.

\section{UNIDAD III:}

1.- Implementación de la Responsabilidad Social. Indicadores y Reportes de Sustentabilidad.

2.- Códigos de ética. Comités de ética.

3.- Casos de organizaciones gestionadas bajo el modelo de RSE.

Objetivos Específicos:

* Conocer la forma en que se operativiza la Responsabilidad Social.

* Tomar contacto con reportes de sustentabilidad y balances sociales emitidos por organizaciones reales.

* Comprender la forma en que las empresas están operando en áreas de Responsabilidad Social, así como los problemas a los que se enfrentan y las oportunidades que genera este modelo de gestión.

\section{UNIDAD IV:}

1.- Responsabilidad Social Universitaria: Concepto, avances en América Latina y en Argentina.

2.- Áreas que abarca la RSU.

3.- Hacia un proyecto de RSU.

Objetivos Específicos:

* Comprender los fundamentos de la RSU.

* Analizar el estado actual de estas prácticas en América Latina y en la Argentina.

* Proponer un proyecto de RSU en los cuales los participantes estén implicados.

\subsection{Metodología de Trabajo}

El dictado del seminario se implementará mediante la modalidad de taller, con exposiciones del docente, dialogadas con los alumnos y actividades grupales de análisis de casos-problemas -reales y de laboratorio-, algunos de ellos mediante asignación de roles, a efectos de comprobar la asimilación de los conocimientos desarrollados, en su faz teórico-práctica. Todas las acciones estarán dirigidas a conseguir desarrollar la capacidad de análisis crítico de los alumnos.

\subsection{Evaluación de los Aprendizajes}

La evaluación diagnóstica se incluirá en el Formulario de Inscripción, la que constará de cinco preguntas simples, para verificar el grado de conocimiento de los inscriptos acerca de las temáticas Responsabilidad Social y Gestión Estratégica.

La evaluación formativa será continua, a través de la participación en las clases, los análisis 
de los casos-problemas propuestos, bajo la modalidad de pequeños equipos de trabajo, de no más de cinco participantes.

Para aprobar este seminario, se deberá entregar un proyecto, de carácter individual o de a pares, de implementación de políticas de RSU en el área, departamento o cátedra donde el docente se desempeñe.

El mencionado proyecto será evaluado a través de los siguientes criterios:

Creatividad y coherencia de la propuesta.

Claridad en la redacción.

Utilidad, calidad y pertinencia del proyecto

La escala de calificaciones se regirá por la Resolución N 473/o8 C.S., vigente para la Universidad Nacional del Nordeste.

\subsection{Duración}

El presente Seminario-Taller tendrá una duración de 60 horas reloj, a desarrollarse los días viernes de 16:00 a 21:00 y sábado de 08:00 a 13:00, durante tres semanas consecutivas.

\section{CONCLUSIONES}

Cuando hablamos de Responsabilidad Social Universitaria nos referimos a conducir a la universidad a observar con ojos críticos su actuación frente a las demandas de la realidad social. "Esa realidad le lleva a cuestionarse con relación a su misión como organismo social, como organización autorizada por la comunidad para gestionar el conocimiento, para favorecer el desarrollo de la ciencia y la tecnología orientada a las problemáticas de los grupos humanos, a mirar su condición ética, expresada en términos de respeto por los otros. La lleva a observar sus valores y principios y la aplicación de esos principios en las funciones de docencia, investigación y extensión, de cara a la comunidad, a sus egresados y a sus propios estamentos académicos". (MARTÍNEZ, R., 2008).

No es otra cosa que ser consecuente en el accionar de las funciones universitarias, para que la realización de sus actividades generen en los docentes, no docentes, investigadores y estudiantes de los programas académicos, sensibilidad por lo social, solidaridad con los más necesitados y responsabilidad por un ejercicio sano, constructivo, transparente y generoso frente a las demandas de la comunidad.

Para comenzar con el proceso de responsabilización social universitaria deberemos partir por la concienciación de los actores sociales universitarios acerca de la relevancia, utilidad y pertinencia del concepto y la necesidad de incluirlo en la tarea diaria de todos y cada uno de ellos.

Es por ello que proponemos un programa para un seminario - taller de sensibilización a la RSU para aquellos que se encuentran encargados de la función formativa como una de las más importantes de las misiones universitarias, aunque comprendemos que el proceso es mucho más complejo y que no se trata sólo de docencia, sino también de extensión, gestión e investigación alineadas con un mismo propósito, cual es la ecología armónica entre la univer- 
sidad y la sociedad en la que se desempeña.

La universidad aporta a este escenario un activo de gran riqueza y de mayores posibilidades para una comunidad, la riqueza disponible en su capital intelectual. "Esta riqueza conforma un activo real, determinante en la construcción del destino de los pueblos, es el capital con el cual se construye con certezas de sostenibilidad, especialmente cuando ese capital se encuentra conformado por hombres y mujeres, ciudadanos y ciudadanas de sólida formación profesional y científica, entusiasmados por la búsqueda de una vida mejor para todos y convencidos de su obligación" (Martínez, R. 2008).

Es que el "Ciudadano" debe ser formado en la vida académica, científica y social propia de las entidades universitarias; si falta esta formación de seres con sensibilidad y pertenencia social en la universidad, emergerán consecuencias desalentadoras, tales como el aprovechamiento del más desarrollado hacia el más débil, del más cultivado intelectualmente al menos informado, "en fin, es origen de inequidad y explotación y estas constituyen a su vez, causa de resentimiento y violencia" (Martínez, R., 2008).

\section{REFERENCIAS BIBLIOGRÁFICAS}

AGENTIA (2010) Información Institucional. Obtenido: 26/04/2010. http://www.agentia. unne.edu.ar/

CEGAE (2010) Información Institucional. Obtenido: 25/04/2010. http://cegae.unne. edu.ar/index.htm

CES (2010) Información Institucional. Obtenido: 27/04/2010. http://ces.unne.edu.ar/

CRUZ DE INNOCENTE, R., FRANCHINI, N. JEREZ, S., DE CASTRO, I., GUALTIERI, D., ZÁRATE, J. ET AL. (2007) Proyecto Capacitación en procesos de gestión empresarial y participación para emprendedores (Informe Final) Resistencia, Chaco: Universidad Nacional del Nordeste, Facultad de Ciencias Económicas.

DE LA CALLE, C., GARCÍA RAMOS, J.M. Y GIMÉNEZ, P. (2007) La formación de la responsabilidad social en la universidad. Revista Complutense de Educación. 182 pp. 47-66.

DE LA CUESTA GONZÁLEZ, M., DE LA CRUZ AYUSO, C., RODRÍGUEZ FERNÁNDEZ, J. (2010) Responsabilidad Social Universitaria. La Coruña, España: Netbiblo, pp. 1 - 30.

MARTÍNEZ, R. (2008) Aplicaciones de la responsabilidad social universitaria en el contexto de formación en Colombia. Educación superior y sociedad. Caracas, Venezue-la, 13 (2), pp. $163-175$. 
REPÚBLICA ARGENTINA, MINISTERIO DE EDUCACIÓN (2006). Manual de Voluntariado Universitario. Obtenido 28/04/2010 en http://www.me.gov.ar/spu/guia_tematica/ VOLUNTARIADO/voluntariado.html

VALLAEYS, F. (2008) La Responsabilidad Social Universitaria: ¿Cómo entenderla para querer practicarla? Obtenido: 09/05/2011 en http://dars.pucp.edu.pe/publicaciones_ documentos/La_RSU_Como_entenderla_para_quererla_y_practicarla.pdf

VALLAEYS, F., DE LA CRUZ, C. y SASIA, P. (2009) Manual de primeros pasos en responsabilización social universitaria. Construyendo ciudadanía en universidades responsables. México DF: McGraw-Hill Interamericana Editores, Banco Interamericano de Desarrollo.

ZARATE, J., CRUZ DE INNOCENTE, R., DE CASTRO, I., JEREZ, S., FRANCHINI, N. y GUALTIERI, J. (2006) Proyecto Capacitación en procesos de gestión empresarial y participación para emprendedores, (Inf. Final) Barranqueras, Chaco: Universidad Nacional del Nordeste, Facultad de Ciencias Económicas.

\section{CURRÍCULUM VITAE}

Abogada, egresada de la Facultad de Derecho y Ciencias Sociales y Políticas de la U.N.N.E. con dedicación al derecho Comercial y Empresario. Candidata a Magíster en Gestión Empresarial en la Facultad de Ciencias Económicas de la U.N.N.E. con proyecto de tesis final aprobdo. Auxiliar Docente de Primera Categoría, por concurso, de la Cátedra Derecho Empresario de la Facultad de Ciencias Económicas de la U.N.N.E. Becaria de Postgrado Tipo I por beca de investigación cofinanciada CONICET/UNNE. Formadora en Responsabilidad Social Empresaria, del Programa de Naciones Unidas para el Desarrollo. Experta Universitaria en Responsabilidad Social Corporativa de la UNED, España.

\section{sandraumumansky@yahoo.com.ar}

